\title{
Alta sospecha de enfermedades metabólicas congénitas en la población de ascendencia judía del Hospital Ángeles Lomas
}

\author{
Ana Carolina Medina Doria, ${ }^{1}$ B Oldak Kovalsky, ${ }^{2}$ M Vela Amieva, ${ }^{3}$ \\ I Ibarra González, ${ }^{4}$ C Fernández Lainez, ${ }^{3}$ David Oldak Skvirsky ${ }^{5}$
}

\section{Resumen}

Antecedentes: La aplicación del tamiz neonatal y su ampliación a diversas enfermedades previamente no identificas por este método constituye un reto que ha dado pie a la aplicación de tecnologías como la plataforma múltiple y la espectrometría de masas en tándem. Se ha reconocido predisposición a dichas patologías en comunidades no estudiadas con anterioridad, como la judía. Su estudio es necesario para la identificación oportuna de dichos padecimientos. Objetivo: Reportar el número de tamices neonatales sospechosos de enfermedades metabólicas congénitas dentro de la población de ascendencia judía del Hospital Ángeles Lomas, comparado con el resto de la población. Material y métodos: Se analizaron todos los tamices neonatales realizados entre enero de 2010 y diciembre de 2014 en el Hospital Ángeles Lomas. Los resultados se consignaron en una base de datos exprofeso tomando los casos sospechosos, analizando el sexo y la etnicidad. Resultados: Se registraron 4,749 tamices metabólicos; 1,425 de padres judíos. Fueron sospechosos 172 tamices; de estos, 27 fueron consistentes en la segunda muestra. De estos últimos, $50 \%$ eran mujeres y $41 \%$ eran hombres, ambos judíos. Conclusión: Se observó una elevada sospecha de enfermedades congénitas

Summary
Background: The application of the neonatal screening
and its extension to diseases not identified by the
classic method constitutes a challenge that has led
to the application of new technologies, such as the
multiple platform and the tandem mass spectrometry. A
predisposition to these pathologies has been identified
in certain communities not previously studied, like the
Jewish one; this makes their study essential for their
timely diagnosis. Objective: To report the number of
neonatal screening assays with suspicious reports for a
congenital metabolic disease in those patients of Jewish
ascent in the Angeles Lomas Hospital, and to compare
it to the rest of the cases. Material and methods: We
analyzed all the neonatal screenings performed between
January 2010 and December 2014 at the Angeles
Lomas Hospital. The suspicious results were recorded
in a database, analyzing sex and ethnicity. Results:
4,749 neonatal screenings were analyzed; 1,425 were of
Jewish ascendant. One hundred seventy-two screenings
were suspicious, but only 27 were consistent in the
second test. Of them, $50 \%$ of the females and $41 \%$ of
the males were Jewish. Conclusion: We observed an
elevated incidence of suspicious cases of congenital

Background: The application of the neonatal screening and its extension to diseases not identified by the classic method constitutes a challenge that has led to the application of new technologies, such as the multiple platform and the tandem mass spectrometry. A predisposition to these pathologies has been identified in certain communities not previously studied, like the timish one, this makes their study essential for their neonatal screening assays with suspicious reports for a congenital metabolic disease in those patients of Jewish ascent in the Angeles Lomas Hospital, and to compare it to the rest of the cases. Material and methods: We analyzed all the neonatal screenings performed between January 2010 and December 2014 at the Angeles Lomas Hospital. The suspicious results were recorded in a database, analyzing sex and ethnicity. Results: 4,749 neonatal screenings were analyzed; 1,425 were of Jewish ascendant. One hundred seventy-two screenings second test. Of them, $50 \%$ of the females and $41 \%$ of the males were Jewish. Conclusion: We observed an

\footnotetext{
${ }^{1}$ Pediatra neonatóloga, Hospital Ángeles Lomas, México.

2 Estudiante de Medicina, Universidad Anáhuac, México Norte, Médico interno de pregrado, Hospital de la Beneficencia Española de la Laguna.

${ }^{3}$ Laboratorio de Errores Innatos del Metabolismo y Tamiz, Instituto Nacional de Pediatría.

${ }^{4}$ Instituto de Investigaciones Biomédicas, UNAM.

${ }^{5}$ Pediatra neonatólogo, Jefe de Neonatología, Hospital Ángeles Lomas.
}

Correspondencia:

Dr. David Oldak Skvirsky

Correo electrónico: droldak@yahoo.com.mx

Aceptado: 10-11-2016.

Este artículo puede ser consultado en versión completa en http://www.medigraphic.com/actamedica 
dentro de la población de ascendencia judía, especialmente en G6PD y MCADD.

Palabras clave: Tamiz neonatal, enfermedades congénitas hereditarias, población judía.

\section{INTRODUCCIÓN}

El primer programa de tamiz neonatal $(\mathrm{TN})$ del mundo nació a principios de la década de los 60 en Massachusetts y se convirtió en el primer esfuerzo organizado de salud pública para identificar de manera presintomática enfermedades congénitas tratables. ${ }^{1,2}$ Dicho TN únicamente incluía la detección de la fenilcetonuria (PKU), una patología monogénica autosómica recesiva poco frecuente. Gradualmente, el TN se expandió para incluir otras enfermedades genéticas y no genéticas, y se fueron implementando programas sistematizados en muchos países. ${ }^{3,4}$ En 1973, Jean Dussault y colaboradores en Québec, ${ }^{5}$ siguiendo el exitoso modelo de prevención de la PKU, diseñaron el TN para hipotiroidismo congénito mediante la cuantificación de las hormonas tiroideas en papel filtro. ${ }^{6}$

En las últimas décadas, hemos presenciado avances exponenciales en el conocimiento de las enfermedades metabólicas congénitas y su tratamiento, y ha ocurrido una rápida expansión de las tecnologías llamadas "de plataforma múltiple" para el TN. La espectrometría de masas en tándem ha revolucionado al TN al realizar en forma simultánea la determinación de más de 50 metabolitos, por lo que se ha convertido en el estándar de oro del tamiz moderno. ${ }^{7,8}$ El TN actual incluye la detección tanto de enfermedades genéticas (como los errores innatos del metabolismo y la fibrosis quística) como de otros defectos al nacimiento, como el hipotiroidismo y la toxoplasmosis congénita, entre otros. ${ }^{4}$

Los errores innatos del metabolismo son un conjunto de padecimientos que se presentan como consecuencia de la actividad insuficiente de una vía metabólica, ya sea por deficiencia puntual en una enzima o por distintos errores que llevan a una vía ineficaz. ${ }^{9}$ Dentro de esta categoría, se incluyen más de 500 enfermedades que por sí solas presentan una baja incidencia; sin embargo, al tomar en cuenta todo el grupo, obtienen una incidencia mayor a 1:1,000 habitantes, de manera que representan un conjunto importante de padecimientos. Detectarlos y tratarlos de manera temprana permite mejorar considerablemente la morbilidad y mortalidad. ${ }^{10}$

En México, a pesar de que el TN fue implementado desde 1973 por el Dr. Velázquez Arellano y se ha incorporado en las normas oficiales mexicanas, NOM-007-SA2-1993, ${ }^{11}$ no existe un consenso de cuáles enfermedades se deben incluir en el TN ampliado. Posteriormente, se publicó la diseases in population with Jewish ancestry, especially for G6PD and MCADD.

Key words: Neonatal screening, congenital hereditary diseases, Jewish population.

NOM-034-SSA2-2002 ${ }^{12}$ (modificada en 2013), en la que se menciona una lista de errores innatos del metabolismo que se deben abarcar en el escrutinio (fenilcetonuria, hiperplasia suprarrenal congénita, galactosemia, fibrosis quística, enfermedad de orina de jarabe de maple, homocistinuria e hipotiroidismo congénito, entre otros); sin embargo, no establece la obligatoriedad de su realización. Actualmente, en la Secretaría de Salud, es obligatorio el TN para hipotiroidismo congénito, fenilcetonuria, hiperplasia suprarrenal congénita, galactosemia y fibrosis quística. ${ }^{13}$

Poco hay escrito sobre la incidencia de estas enfermedades en México, ${ }^{14}$ a pesar de que se conocen algunos riesgos para la presencia de las mismas, como es el sexo femenino para el hipotiroidismo congénito, ${ }^{15}$ pertenecer a ciertas comunidades endogámicas o con efecto fundador, como la población judía; ${ }^{16-18}$ sin embargo, hasta nuestro conocimiento no existe ningún estudio en México que analice la frecuencia de estas enfermedades congénitas hereditarias entre la población de ascendencia judía.

El objetivo de este trabajo es reportar el número de tamices neonatales sospechosos de enfermedades metabólicas congénitas dentro de la población de ascendencia judía del Hospital Ángeles Lomas, comparado con el resto de la población tamizada.

\section{MATERIAL Y MÉTODOS}

Se analizaron todos los resultados del TN de los niños nacidos en el Hospital Ángeles Lomas de enero de 2010 a diciembre de 2014, excluyéndose los reingresos al área y los pacientes trasladados de otros hospitales.

El estudio se hizo previo consentimiento verbal informado, con fundamento en la normatividad mexicana vigente. ${ }^{13} \mathrm{~A}$ todos los recién nacidos mayores de 48 horas, se les realizó al momento del egreso hospitalario una punción con lanceta estéril en el talón y se recolectaron cinco gotas de sangre en papel filtro (tarjeta de Guthrie).

A los menores de 48 horas de vida se les citó para que acudieran a hacer el TN antes de los 10 días de vida. El procesamiento del TN se realizó en el Hospital Ángeles Pedregal y los resultados se consignaron en una base de datos exprofeso.

Se analizó el número de tamices sospechosos; es decir, todos aquellos casos en los que el resultado obtenido fue 
anormal tomando como referencia el valor de corte establecido por el laboratorio. Todos los casos sospechosos se localizaron en la base de datos y se analizó el sexo del producto y la ascendencia étnica referida por los padres.

\section{RESULTADOS}

En el periodo comprendido entre enero de 2010 y diciembre de 2014 se registraron 5,069 nacimientos en el área de neonatología del Hospital Ángeles Lomas. De estos, se tomaron 4,749 tamices metabólicos, lo cual indica una cobertura de $93.69 \%$. Un total de 1,425 recién nacidos fue de padres judíos y 3,324 de otro origen étnico. Se encontraron 172 tamices sospechosos ( 1 de cada 28) en la población general analizada. De estos, 27 casos fueron consistentemente anormales en la segunda muestra (uno de cada 176). En este subgrupo de 27 recién nacidos altamente sospechosos, se analizó la ascendencia y se encontró que 12 eran judíos (1:119) y 15 tenían otro origen étnico (1:222).

La frecuencia de enfermedades sospechadas según la ascendencia se muestra en el cuadro 1.

De los 27 casos sospechosos, se encontraron 10 niñas y 17 niños, de los cuales 5/10 (50\%) eran judías y 7/17 (41\%) fueron judíos de sexo masculino.

\section{DISCUSIÓN}

El resultado más notable de esta investigación, debido a que es el primer estudio en México que reporta el comportamiento de subpoblaciones en cuanto a errores innatos del metabolismo, es la elevada proporción de sospechas de enfermedades congénitas hereditarias dentro de la población de recién nacidos de ascendencia judía, la cual fue de $0.84 \%$, comparada con $0.45 \%$ de la población de otro origen étnico (Cuadro 1). Esta elevada proporción de sospechas está fundamentalmente dada por el alto número de sospechas de G6PD y MCADD encontrado dentro de la población judía $(0.42 \%$ y $0.14 \%$, respectivamente, cuadro 1). Es muy conocido que la deficiencia de G6PD es más frecuente dentro de la población mediterránea, africana y asiática, y que la alta prevalencia de heterocigotos se relaciona con una ventaja evolutiva contra la malaria. ${ }^{19} \mathrm{En}$ la población judía estudiada en el presente trabajo, muy seguramente existe una mayor proporción del subgrupo de judíos sefaraditas, que pudiesen provenir del mediterráneo, lo que podría explicar la elevada frecuencia de sospechas de deficiencia de G6PD.

La principal limitante de esta investigación es que se consideró a todos los recién nacidos de ascendencia judía en una sola categoría, y es conocido que existen diversos subgrupos poblacionales, tales como Ashkenazim, Sefaradim, o bien, hijos de matrimonios mixtos (padre o madre

\begin{tabular}{|c|c|c|}
\hline \multicolumn{3}{|c|}{ Cuadro 1.} \\
\hline $\begin{array}{l}\text { Enfermedad } \\
\text { sospechada }\end{array}$ & $\begin{array}{c}\text { Judíos } \\
n=1,425\end{array}$ & $\begin{array}{l}\text { No judíos } \\
n=3,324\end{array}$ \\
\hline Deficiencia de G6PD & $6(0.42 \%)$ & $5(0.15 \%)$ \\
\hline Deficiencia de MCADD & $2(0.14 \%)$ & $1(0.03 \%)$ \\
\hline $\begin{array}{l}\text { Hiperplasia suprarrenal } \\
\text { congénita }\end{array}$ & $1(0.07 \%)$ & $2(0.6 \%)$ \\
\hline Hiperfenilalaninemia & $1(0.07 \%)$ & $1(0.03 \%)$ \\
\hline Hipotiroidismo & $1(0.07 \%)$ & $3(0.09 \%)$ \\
\hline Tirosinemia transitoria & $1(0.07 \%)$ & $1(0.03 \%)$ \\
\hline Galactosemia & 0 & $1(0.03 \%)$ \\
\hline Hemoglobinopatías & 0 & $1(0.03 \%)$ \\
\hline Total & $12(0.84 \%)$ & $15(0.45 \%)$ \\
\hline
\end{tabular}

judíos con pareja de otro origen), por lo que sería interesante ampliar este trabajo considerando dichas variables.

Es de notar que la deficiencia de la deshidrogenasa de Acil CoA de cadena media (MCADD por sus siglas en inglés) también tiene una distribución poblacional característica, siendo más frecuente en poblaciones caucásicas y de Medio Oriente que en las hispanas. ${ }^{20}$

Por otro lado, el TN del Hospital Ángeles Lomas tiene una cobertura aceptable de $93.69 \%$, semejante a la reportada en otros programas; ${ }^{14,21}$ sin embargo, esto señala que el $6.31 \%$ de los recién nacidos en la institución no son tamizados por diversas causas, entre las que destacan el rechazo del estudio por el costo y el alta temprana, principalmente.

Son pocos los trabajos en el país que describen incidencias de enfermedades metabólicas. Este artículo aporta conocimientos útiles para que el TN se realice en todos los recién nacidos en México, y muy especialmente en aquellos de ascendencia judía.

Por otro lado, de acuerdo con nuestro estudio, sería recomendable incorporar la detección de deficiencia de glucosa 6 fosfato-deshidrogenasa dentro del tamiz básico que se realiza en México y que sólo incluye hipotiroidismo congénito, galactosemia, fenilcetonuria, hiperplasia suprarrenal congénita $y$, recientemente, fibrosis quística.

Si bien en México la población es una mezcla muy compleja de grupos de variados orígenes, ${ }^{22}$ los habitantes de ascendencia judía también son genéticamente diversos.

Finalmente, es importante mencionar que en esta investigación no se pudo conocer el número total de casos confirmados debido a que, por las características de la institución, no existe una base de datos general en la que 
dichos casos se registren y su seguimiento se conozca. Es importante solventar este problema para tener un mejor conocimiento sobre la epidemiología de las enfermedades metabólicas congénitas que afectan a los recién nacidos en el Hospital Ángeles Lomas y, con ello, planear adecuadamente y optimizar el programa de TN.

\section{Agradecimientos}

Agradecemos a Margarita Cañada Ledesma, jefa del Laboratorio Clínico del Hospital Ángeles Lomas, por su apoyo para la realización de este proyecto.

\section{REFERENCIAS}

1. Scriver CC. A simple phenylalanine method for detecting phenylketonuria in large populations of newborn infants, by Robert Guthrie and Ada Susi. Pediatrics. 1963; 32: 338-343.

2. Natowicz M. Newborn screening — setting evidence-based policy for protection. N Engl J Med. 2005; 353 (9): 867-870.

3. Berry SA. Newborn screening. Clin Perinatol. 2015; 42: 441-453.

4. Therrell BL, Padilla CD, Loeber JG, Kneisser I, Saadallah A. Borrajo JC et al. Current status of newborn screening worldwide: 2015. Semmin Perinatol. 2015; 39: 171-187.

5. Dussault JH. The anecdotal history of screening for congenital hypothyroidism. J Clin Endocrinol Metab. 1999; 84: 4332-4334.

6. Delange F. Neonatal screening for congenital hypothyroidism: results and perspectives. Horm Res. 1997; 48: 51-61.

7. AAP Newborn Screening Task Force. Saving the family from birth to the medical home: newborn screening: a blueprint for the future -a call for a national agenda on state newborn screening programs. Pediatrics. 2000; 106: 389-422.

8. Fernández-Lainez C, Aguilar-Lemus JJ, Vela-Amieva M, IbarraGonzález I. Tandem mass spectrometry newborn screening for inborn errors of intermediary metabolism: abnormal profile interpretation. Curr Med Chem. 2012; 19: 4511-4522.

9. Ibarra-González I, Fernández-Lainez C, Belmont-Martínez L, Guillén-López S, Monroy-Santoyo S, Vela-Amieva M. Caracterización de errores innatos del metabolismo intermediario en pacientes mexicanos. An Pediatr (Barc). 2014; 80: 310-316.
10. Raise GM, Steiner RD. Inborn errors of metabolism (metabolic disorders). Pediatr Rev. 2016; 37: 3-15.

11. Norma Oficial Mexicana NOM-007-SA2-1993. Atención de la mujer durante el embarazo, parto y puerperio y recién nacido. Criterios y procedimientos para la prestación del servicio. Diario Oficial de la Federación, órgano del Gobierno Constitucional de los Estados Unidos Mexicanos, tomo CDXCDI; 1995.

12. Norma Oficial Mexicana NOM-034-SSA2-2002. Para la prevención y control de los defectos al nacimiento. Diario Oficial de la Federación, órgano del Gobierno Constitucional de los Estados Unidos Mexicanos, tomo DLXXVII; 2003.

13. Norma Oficial Mexicana NOM-034-SSA2-2013. Para la prevención y control de los defectos al nacimiento. Diario Oficial de la Federación, órgano del Gobierno Constitucional de los Estados Unidos Mexicanos, tomo. DCCXXXII; 2014.

14. Trigo-Madrid M, Díaz-Gallardo J, Mar-Aldana R, Ruiz-Ochoa D, Moreno-Graciano C, Martínez-Cruz P. Resultados del Programa de Tamiz Neonatal Ampliado y epidemiología perinatal en los servicios de sanidad de la Secretaría de Marina Armada de México. Acta Pediátr Mex. 2014; 35: 448-458.

15. Monroy-Santoyo S, Ibarra-González I, Fernández-Lainez C, Greenawalt-Rodríguez S, Chacón-Rey J, Calzada-León R et al. Higher incidence of thyroid agenesis in Mexican newborns with congenital hypothyroidism associated with birth defects. Early Hum Dev. 2012; 88: 61-64.

16. Al-Thihli K, Al-Murshedi F, Al-Hashmi. N, Al-Mamari W, Islam MM, Al-Yahyaee SA. Consanguinity, endogamy and inborn errors of metabolism in Oman: A cross sectional study. Hum Hered. 2014; 77: 183-188.

17. Laberge AM, Michaud J, Richter A, Lemyre E, Lambert M, Brais B et al. Population history and its impact on medical genetics in Quebec. Clin Genet. 2005; 68: 287-301.

18. Klugman S, Gross SJ. Ashkenazi Jewish screening in the twenty-first century. Obstet Ginecol Clin North Am. 2010; 37: 37-46.

19. Luzzato L, Usanga EA, Reddy S. Glucose-6-phosphate dehydrogenase deficient red cells: Resistance to infection by malarial parasites. Science. 1969; 164: 839-842.

20. Feuchbaum L, Carter J, Dowray S, Currier RJ, Lorey F. Birth prevalence of disorders detectable through newborn screening by race/ethnicity. Genet Med. 2012; 14: 937-945.

21. Borrajo GJ. Newborn screening in Latin America at the beginning of the 21st century. J Inherit Metab Dis. 2007; 30: 466-481.

22. Moreno-Estrada A, Gignoux CR, Fernández-López JC, Zakharia F, Sikora M, Contreras AV et al. Human genetics. The genetics of Mexico recapitulates native American substructure and affects biomedical traits. Science. 2014; 344: 1280-1285. 